\title{
Macrolides for the treatment of chronic bacterial prostatitis: An effective application of their unique pharmacokinetic and pharmacodynamic profile (Review)
}

\author{
G. PERLETTI ${ }^{1 *}$, V.SKERK ${ }^{2 *}$, V. MAGRI ${ }^{3}$, A. MARKOTIC $^{2}$, S. MAZZOLI $^{4}$, \\ M.J. PARNHAM ${ }^{2}$, F.M.E. WAGENLEHNER ${ }^{5}$ and K.G. NABER ${ }^{6}$
}

\begin{abstract}
${ }^{1}$ Department of Biomedical, Inf., Comm. and Env. Sciences, University of Insubria, Busto A, I-21052 Varese, Italy; ${ }^{2}$ University Hospital for Infective Diseases 'Dr Fran Mihaljević', HR-10000 Zagreb, Croatia; ${ }^{3}$ Urology and Prostatitis Secondary Care Clinic, Hospital 'Istituti Clinici di Perfezionamento', Milan; ${ }^{4}$ STDs Centre, S.M. Annunziata Hospital, Florence, Italy; ${ }^{5}$ Justus Liebig-University, Department of Urology, Pediatric Urology and Andrology, Giessen;

${ }^{6}$ Technical University Munich, Munich, Germany
\end{abstract}

Received July 7, 2011; Accepted August 2, 2011

DOI: $10.3892 / \mathrm{mmr} .2011 .575$

\begin{abstract}
Chronic bacterial prostatitis (CBP) is a persistent infection of the prostate characterized by poor quality of life mainly due to frequent relapse episodes caused by incomplete eradication of causative pathogens. Aggressive antibacterial therapy is required to attenuate the severe symptoms of CBP and to achieve a permanent cure. Although fluoroquinolones are currently recommended as first-choice agents, macrolide antibiotics are emerging as a noteworthy option for the treatment of CBP. Macrolide antibiotics are characterized by an impressive array of distinct pharmacokinetic (PK) and pharmacodynamic (PD) properties. These properties include high intracellular accumulation in phagocytes and at sites of infection, including the prostate; broad antibiotic but also biofilm-inhibiting properties; immunomodulating and inflammation-resolving activities. These features offer particular advantages for the treatment of chronic infections of the prostate gland, which are not easily amenable to drug therapy. Macrolides may be exploited to counteract the unsatisfactory rates of clinical symptom improvement and pathogen eradication. The results of a number of clinical trials support this proposal.
\end{abstract}

\section{Contents}

1. Prostatitis: definition, clinical implications and mechanistic issues

Correspondence to: Dr Gianpaolo Perletti, Dipartimento di Biologia Strutturale e Funzionale, Università degli Studi dell'Insubria, Via A. Da Giussano 12, Busto A., I-21052 Varese, Italy

E-mail: gianpaolo.perletti@uninsubria.it

*Contributed equally

Key words: prostatitis, macrolides, azithromycin, biofilm, Chlamydia trachomatis, urological infections, sexually-transmitted infections
2. Treatment options for chronic bacterial prostatitis

3. Optimal pharmacokinetic characteristics of antibacterial agents in the prostate. Pharmacokinetic and pharmacokinetic/pharmacodynamics of macrolides

4. Pharmacodynamics of macrolides: structure, antibacterial activity, resistance issues

5. Pharmacodynamics of macrolides: non-antibiotic pharmacological properties

6. Macrolide administration: safety issues

7. Two pivotal macrolide targets in the prostate gland: intracellular bacteria and biofilms

8. Clinical evidence of efficacy of macrolides in prostate infections: monotherapy

9. Clinical evidence of efficacy of macrolides in prostate infections: combination therapy

10. Conclusions

1. Prostatitis: definition, clinical implications and mechanistic issues

Human prostate pathologies, such as adenocarcinoma and inflammatory conditions of the gland, are among the most challenging clinical problems of the third millennium. In particular, inflammation of the prostate gland is showing a marked increase in males between the ages of 20 and 40 . This increase has a considerable impact in terms of social, health-related and individual costs, and a pronounced effect on fertility and patient quality of life. Chronic prostatitis (CP) is a major emerging problem in males of fertile age. The main $\mathrm{CP}$ characteristics are the early onset of symptoms, their persistence for years, the relatively young age of patients, the possible impact of sexually-transmitted infections acquired during the first sexual intercourses, and the diverse and severe long-term sequelae (e.g., infertility). A multicentre, cohort, observational study conducted in Italy on 750 male patients between the ages of 25 and 50 years with symptoms of $\mathrm{CP} /$ chronic pelvic pain syndrome (CPPS), revealed a relatively high prevalence of 
prostatitis (13.3\%), probably due to the application of systematic diagnostic criteria (Meares-Stamey test and urethral swab) to all patients (1). In 1995, the USA National Institutes of Health (NIH) defined a new prostatitis classification system, which divides prostatitis syndromes into acute and chronic, bacterial and abacterial, symptomatic and asymptomatic. It is commonly known that only $5-10 \%$ of CP patients have a bacterial infection detectable by conventional cultures and only a small fraction of patients presenting with chronic prostatitis have evidence of infection caused by commonly recognized uropathogens.

According to the NIH definition, chronic bacterial prostatitis (CBP, Category II, NIH-NIDDK) is diagnosed when pathogens are cultured from a chronically infected prostate in patients suffering from repeated symptomatic episodes of bacteriuria (2). The syndrome is characterized by an array of signs and symptoms, including perineal pain, testicular pain and/or other forms of pelvic pain, irritative and/or obstructive bladder symptoms, haematuria, pyuria, ejaculatory pain and sexual dysfunction. Since CBP signs and symptoms frequently overlap with the features of NIH-category III ('abacterial', 3) $\mathrm{CP} / \mathrm{CPPS}$, the latter is mainly diagnosed by excluding bacterial infection in symptomatic patients.

$\mathrm{CP}$ syndromes are serious, invaliding conditions. The quality of life for a man suffering from $\mathrm{CP}$ is similar to that experienced by patients with myocardial infarction, unstable angina, or Crohn's disease (4).

The California Men's Health Study has shown an association of CP with prostate cancer. This prospective study, performed on a sample of about 70,000 patients, showed that male individuals who reported a history of prostatitis had a $30 \%$ higher chance of prostate cancer diagnosis $(\mathrm{R}=1.30$; 95\% CI, 1.10-1.54). Those individuals who had prostatitis for more than one year had an increased risk of prostate cancer $(\mathrm{RR}=1.50 ; 95 \% \mathrm{CI}, 1.08-2.08)$ (5). The role of inflammation as a potential risk factor for prostate cancer (PCa) is indirectly supported by the finding that chronic NSAID intake is capable of decreasing the risk for $\mathrm{PCa}(6,7)$, and by the observations that polymorphisms in the gene coding the anti-inflammatory cytokine interleukin-10 (IL-10) are correlated to PCa aggressiveness as well as to progression to advanced stage PCa (8).

These findings suggest that aggressive and correct treatment of CP is not only essential for relieving and mitigating the severe symptoms of the disease, but may actually convey additional important benefits in terms of personal and public health.

Despite the increasing number of preclinical and clinical studies focusing on prostate inflammatory diseases, little is known about the immunobiology of the prostate. Most studies on prostate inflammation and immunity are based on in vitro or animal models, and relatively few data exist pertaining to patients affected by various diseases of the prostate gland. Recent findings emphasize the important role of prostate epithelial cells in the production of mediators of inflammation, as well as their active role in the mechanisms of non-specific immunity during acute and chronic infection and in the presence of infection-induced inflammatory conditions (9).

Initial results suggest that a significant role in acute prostatitis and CP is played by Toll-like receptors (TLRs) such as TLR-4. TLRs are important modulators of reactions involved in non-specific immunity. In particular, these receptors play a crucial role in allowing prostate epithelial cells to recognize microorganisms, and in turn to initiate mechanisms of non-specific immunity as a first line of defense in bacterial prostatitis (10-12). In addition to epithelial cells, specific and non-specific immunity in diseases of the prostate is mediated by various mediator-secreting inflammatory cells that may generate signals directing immune reactions to acute prostatitis or CP (13). These mechanisms have wide relevance in that $\mathrm{CP}$ increases the risk of prostate cancer and autoimmune disorders may lead to autoimmune prostatitis. Thus, thorough and extensive investigation is required, not only for a better understanding of the immune reactions that occur during various diseases of the prostate, but also in order to reveal improved approaches to drug therapy. In this respect, the growing appreciation of the immunomodulatory effects of macrolide antibiotics offers considerable potential for the treatment of acute and chronic prostatitis.

\section{Treatment options for chronic bacterial prostatitis}

Treatment of category II CBP primarily involves the use of antibacterial agents to eliminate the organisms producing the infection. In addition, pain and inflammation are frequently treated with NSAIDs, whereas treatment of voiding symptoms is often based on administration of $\alpha$-adrenoceptor blockers $(14,15)$.

There is broad consensus for the use of fluoroquinolones as first-choice antibacterial agents for the treatment of category II CBP. International guidelines recommend a 4-12 week course of ciprofloxacin, lomefloxacin, ofloxacin, levofloxacin or norfloxacin for the eradication of susceptible pathogens $(16,14)$.

Introduction of these drugs has greatly improved the outcome and outlook for CBP patients, achieving pathogen eradication rates of over $80 \%$ (17). However, permanent cure of the infection is frequently hampered by relapse caused by incomplete pathogen eradication (18). This restriction is likely due to the sub-optimal prostatic concentrations of most quinolones or to the transition of planctonic pathogens to sessile, quorum-sensing-activated, bacterial chemoresistant biofilms.

Trimethoprim, whether or not combined with sulfamethoxazole, was formerly the best available treatment option for CBP), achieving pathogen eradication in approximately $30-60 \%$ of cases [reviewed in (19)]. This drug is now indicated as a last-resource agent in case of fluoroquinolone resistance or when first-choice agents are contraindicated (16).

Macrolides have currently received limited attention for the treatment of CBP, and their use in Europe is currently restricted to 'special indications', without detailed specification (16).

The fact that macrolides are a treatment option worthy of greater attention was emphasized by a panel of experts at an international CP and CPPS syndrome symposium held in the United Kingdom in 2008 (20). In that context, macrolides, such as azithromycin and erythromycin, were recommended as second-choice agents after fluoroquinolones, as they have higher cure rates for Chlamydia than ciprofloxacin, the highest cure rates in vitro against mycoplasmata, distribute optimally to the prostate and may penetrate and inhibit biofilms (20).

Lipsky and co-workers have reviewed the evidence supporting the use of macrolides in CBP and suggested 
Table I. Median concentrations of fluoroquinolones or macrolides in PS or PT and the ratios of their concentrations in either PS or PT to those in PL in healthy volunteers or prostatitis patients (where possible normalised to a dose of $400 \mathrm{mg}$, assuming linear $\mathrm{PKs})$.

\begin{tabular}{|c|c|c|c|c|c|c|c|}
\hline Antibiotic & Dosage & Subjects (no.) & $\begin{array}{l}\text { Hours from } \\
\text { last dose }\end{array}$ & $\begin{array}{c}\mathrm{PS} \\
(\mathrm{mg} / \mathrm{l})\end{array}$ & $\begin{array}{c}\mathrm{PS} / \mathrm{PL} \\
\text { ratio }\end{array}$ & $\begin{array}{c}\mathrm{PT} \\
(\mathrm{mg} / \mathrm{l})\end{array}$ & $\begin{array}{l}\mathrm{PT} / \mathrm{PL} \\
\text { ratio }\end{array}$ \\
\hline Norfloxacin & 400 mg normalised SD (p.o.) & 7 & $1-4$ & 0.08 & 0.10 & & \\
\hline Ciprofloxacin & 400 mg normalised SD (i.v.) & 8 & 4 & 0.18 & 0.20 & & \\
\hline Fleroxacin & 400 mg normalised SD (p.o.) & 8 & $2-4$ & 1.00 & 0.28 & & \\
\hline Levofloxacin & 400 mg normalised SD (p.o.) & 8 & 3 & 1.42 & 0.29 & & \\
\hline Ofloxacin & 400 mg normalised SD (i.v.) & 5 & 4 & 0.66 & 0.33 & & \\
\hline Enoxacin & 400 mg normalised SD (p.o.) & 10 & $2-4$ & 0.29 & 0.39 & & \\
\hline Lomefloxacin & 400 mg normalised SD (p.o.) & 7 & 4 & 1.38 & 0.48 & & \\
\hline Gatifloxacin & 400 mg normalised SD (p.o.) & 7 & 4 & 1.03 & 1.29 & & \\
\hline Moxifloxacin & 400 mg normalised SD (p.o.) & 8 & $3-4$ & 3.99 & 1.57 & & \\
\hline Rosamicin & $1000 \mathrm{mg} / \mathrm{d}$ steady state (p.o.) & 1 & 3 & 19.00 & 211 & & \\
\hline Erythromycin & 1000 mg/d steady state (p.o.) & 2 & 3 & 0.41 & 1.50 & & \\
\hline Clarithromycin & 750 mg/d steady state (p.o.) & 13 & 7 & & & 3.83 & 2.54 \\
\hline Azithromycin & 2x250 mg (p.o.) & 14 & 15 & & & 2.54 & $\sim 20$ \\
\hline Erythromycin & $1000 \mathrm{mg} / \mathrm{d}$ steady state (p.o.) & 9 & 2 & & & 0.58 & 0.90 \\
\hline
\end{tabular}

PS, prostatic secretions; PT, prostate tissue; PL, plasma; PK, pharmacokinetic; p.o., per oral; i.v., intravenous; SD, single dose.

their administration within the frame of various therapeutic schemes (21). Notably, Jiang et al have recently described the resolution with azithromycin of a difficult case of multiresistant $E$. coli CBP (22), suggesting that macrolides possess unexplored and unexpected activities.

3. Optimal pharmacokinetic (PK) characteristics of antibacterial agents in the prostate. $\mathrm{PK}$ and $\mathrm{PK} / \mathrm{pharmaco}$ dynamic (PD) properties of macrolides

For effective antimicrobial therapy, the pathogens at the site of infection should be exposed to drug concentrations sufficiently high to inhibit bacterial growth or even to eradicate the causative agents from that site. Considerable evidence suggests that bacteria in the prostatic tissue survives in a milieu protected by biofilms (23). Therefore, antimicrobial agents that are active in biofilms, such as fluoroquinolones and macrolides, may be preferred drugs in CBP (24). The prostate exhibits several anatomical barriers for the distribution of antimicrobial molecules: i) prostate glands are perfused by non-fenestrated capillaries; ii) the prostatic epithelium apparently lacks active transport mechanisms facilitating drug uptake; iii) antibiotics should travel across capillary and prostatic epithelia to reach prostatic secretions (PS) and prostatic ducts, i.e., the primary site of infection in CBP (25).

Three factors are important for the PK behavior of drugs in the prostate: serum protein binding, lipid solubility and $\mathrm{pH}$-partition (26). PK studies investigating the distribution of antibiotics in prostate secretions have been performed in animals, dogs in particular, and in healthy volunteers, whereas studies investigating the prostate tissue (PT) concentrations of the same agents have mainly been performed in patients (27). Data from studies in dogs indicate a drawback of the acidic $\mathrm{pH}$ of dog PS, which is not observed in healthy male individuals, in whom prostate $\mathrm{pH}$ mainly resembles the plasma $\mathrm{pH}$. In patients with active chronic infection of the prostate, the $\mathrm{pH}$ may even reach alkaline values. An additional drawback of available PK studies is indicated by the fact that PTs collected from patients contain a mixture of different cells and fluids from various compartments of the gland (blood cells, serum, interstitial fluid, fibromuscular stroma, secretory epithelium, nerve tissue and vascular tissue) and may not represent the precise site of infection in CBP. Therefore, an ideal PK model has yet to be produced in clinical practice, although assessing PKs in PS appears to be a more accurate strategy.

Most PK studies have been performed with fluoroquinolones. However, the results of these studies should be interpreted with caution, unless care was taken to rule out or to at least identify urinary/urethral contamination. By analysing the concentrations of various fluoroquinolones in prostatic and seminal fluid as well as in PT, it became obvious that fluoroquinolones differ, not only in their bioavailability, but also in their ability to penetrate into these sites (24). The prostatic fluid-to-plasma concentration ratios of various fluoroquinolones, measured in healthy volunteers, showed a broad range of prostatic-fluid-to-plasma ratios $(0.10-1.57)$ (Table I) $(27,28)$. In studies in which fluoroquinolone concentrations were examined in PT obtained at transurethral resection, the antibiotics appeared to be present consistently at or above corresponding plasma concentrations, which may be explained by the heterogeneous material investigated with this methodology.

Few studies in which the kinetics of macrolides in the prostate have been investigated showed that macrolides penetrate well into PT and prostatic and seminal fluids (Table I) (29). For example, the mean maximum concentrations of azithromycin and clarithromycin in human prostatic tissue 
after 500 or $750 \mathrm{mg}$ oral dosing were 2.54 and $3.83 \mu \mathrm{g} / \mathrm{ml}$, respectively, and were higher than plasma concentrations (about 20- and 2-fold, respectively) (29,30). Rosamicin, another macrolide with gram-negative activity, was investigated in dogs with normal and infected prostates and compared to erythromycin (31). High PT and fluid to plasma ratios were found. Prostatic fluid to plasma ratios of rosamicin were significantly higher than those for erythromycin in patients (tissue/plasma, 32-fold; fluid/plasma, 100-fold) (31).

Since CBP occurs most frequently in a biofilm state, the addition of a macrolide as a highly effective agent against biofilms may theoretically translate into improved microbiological eradication, although this has yet to be proven. The enhanced PK properties of newer generation antibiotics, with regard to prostate penetration, have considerably improved patient outcomes compared to the older substances $(24,27)$. Therefore, the selection of an appropriate antimicrobial agent that has optimal PK properties for PS and PT is of paramount importance for the treatment of CBP.

In this respect, azithromycin shows noteworthy characteristics. It has a large volume of distribution $(231 / \mathrm{kg})$, and low peak serum levels $(0.4 \mu \mathrm{g} / \mathrm{ml})$, properties that are consistent with extensive tissue distribution and high intracellular (lysosomal) accumulation of the drug (32-34).

Metabolism of macrolides is predominantly hepatic, biliary excretion being a major pathway of elimination. The plasma half-life of azithromycin is markedly long ( about $70 \mathrm{~h}$ ). This important feature, combined with extensive distribution and high tissue concentrations, allows drug concentrations to remain above the MICs for the whole-dosing interval, if administered once-daily, in most clinical situations $(35,36)$. It was found that a $1.5 \mathrm{~g}$ dose of azithromycin was administered for 3 or 5 consecutive days, without notable changes in efficacy (37). For this reason, as discussed in the last section of this article, azithromycin has been administered to CBP patients by different investigators in the form of a 'pulsedosing' regimen for 3 consecutive days weekly, for a varying number of weeks.

PK/PD properties of macrolides are markedly diverse, depending on the specific agent considered. Whereas the $\mathrm{AUC}_{24 \mathrm{~h}} / \mathrm{MIC}$ is the primary determinant for the clinical efficacy of azithromycin, the time above MIC is the most crucial PK/PD parameter for optimal dosing of erythromycin $(36,38)$. It is worth mentioning that the antibacterial activity of macrolides is markedly affected by an acidic milieu, as over $90 \%$ of activity is lost for each 1 unit decrease in $\mathrm{pH}$ (36).

\section{Pharmacodynamics of macrolides: structure, antibacterial activity, resistance issues}

In 1949, the scientist Abelardo Aguilar, sent his employer, Eli Lilly Co., samples of an antibiotic isolated from a soil that he had collected in the central Philippines. The soil contained the strain Saccharopolyspora erythraea, which proved to be the source of a new antibiotic, known as 'Ilosone', in honour of the Iloilo province, where the soil had been sampled. It was the first successful macrolide antibiotic and was introduced to the USA market in 1952. Its broad antimicrobial spectrum provided an alternative for patients showing allergic reactions to penicillin, or who were infected with penicillin-resistant
Staphylococcus aureus. This was a time at which penicillinaseexpressing strains were starting to spread around the world.

Macrolides are a group of antibacterial agents belonging to the polyketide class of natural antibiotics. These agents are structurally characterized by a large macrocyclic lactone, the macrolide ring (Fig. 1). The lactone rings of macrolides are usually 14-, 15- or 16-membered.

Erythromycin, a 14-membered ring antibiotic, was the first macrolide to be adopted in medical therapy. Drug bioavailability problems, caused by instability of the drug in an acidic milieu, prompted the design of newer analogues, characterized by increased gastric stability and broader activity spectrum. Newer generations of macrolides included 14-membered ring agents such as the closely related agent clarithromycin (6-0methylerythromycin), the 15 -membered ring azalide azithromycin (39), and the 16-membered ring josamycin.

Two deoxy-sugars, usually desosamine and cladinose, are attached to the macrolide ring (Fig. 1). The latter [(4R,5S,6S)4-methoxy-4,6-dimethyl-tetrahydropyran-2,5-diol] is essential for the antibacterial activity of 14- or 15-membered macrolides; in the absence of other modifications, removal of this sugar leads to loss of antimicrobial activity (40).

Macrolides bind to domains II and V of the large 50S ribosomal subunit inside the nascent peptide exit tunnel, formed primarily by $23 \mathrm{~S}$ rRNA, in the vicinity of the peptidyl transferase centre, and arrest bacterial cell growth by inhibiting protein synthesis. The exact mechanism of macrolide inhibition of ribosome activity is poorly understood (41).

Macrolides inhibit protein synthesis in a fairly wide spectrum of micro-organisms and are indicated for conditions including upper and lower respiratory tract infections, otitis media, skin/soft tissue infections and sexually-transmitted urogenital infections caused by intracellular pathogens or Gonococci. Azithromycin is also an emerging antimalarial agent (42).

The spectrum of activity of macrolides such as azithromycin includes a variety of gram-positive and -negative pathogens, such as Streptococcus pneumoniae, Staphylococcus aureus, Listeria monocytogenes, Neisseria spp., Moraxella catharralis, Haemophylus influenzae, Salmonella and Sighella spp., Legionella spp., Chlamydia trachomatis, Mycoplasmata, Mycobacterium avium, as well as anaerobes such as Actinomyces spp., Clostridium spp. and Peptostreptococci and some fungi (43).

The most frequently found resistance mechanism to macrolides is the modification of the drug binding site by dimethylation of a single 23S rRNA nucleotide, A2058, by constitutively expressed or inducible Erm-type methyltransferases. This modification markedly decreases the affinity of macrolides for their target. The same resistant phenotype can be attained by base substitutions at position 2058 (44). Besides macrolides, other antibacterial agents such as lincosamides and streptogramin show decreased affinity for the mutant $23 \mathrm{~S}$ rRNA (41).

In addition to target site methylation, macrolide resistance may be mediated by the expression of minigene-coded oligopeptides of 4-6 aminoacids, similar to erm leader peptides, and is likely to act to make ribosomes refractory to inhibition by macrolides. For example, oligopeptides responsible for clarithromycin resistance are MLLLV, MLLVV and MVLMV, 

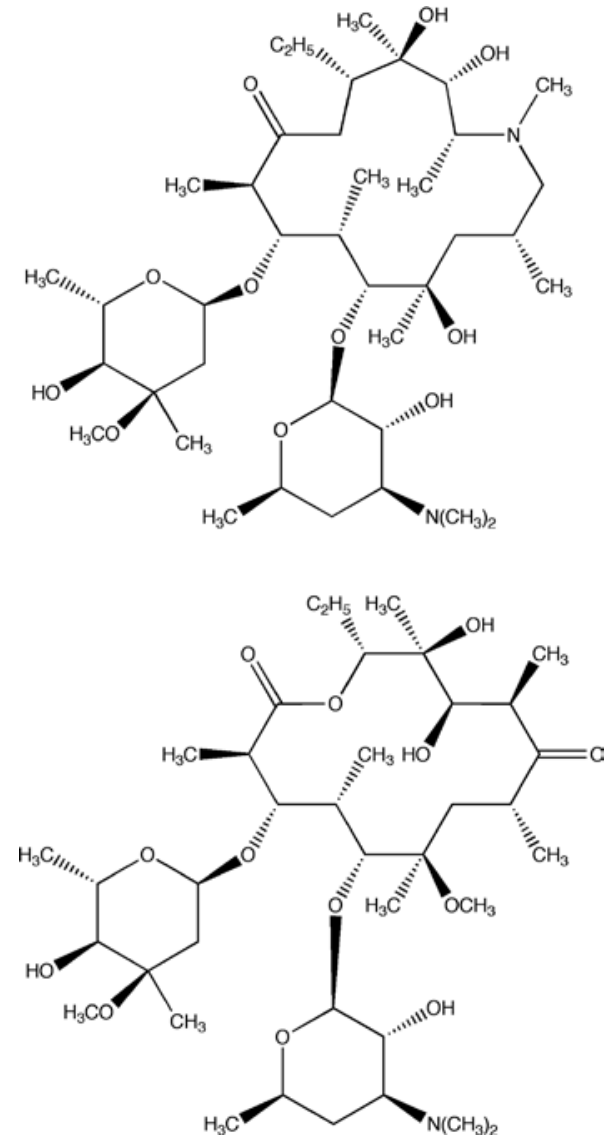

Figure 1. Molecular structure of two representative macrolides: the 14-membered clarithromycin (bottom) and the 15-membered azithromycin (top)

among others [reviewed in (45)]. Other resistance mechanisms include the production of drug-inactivating esterases or kinases or the expression of efflux proteins.

\section{Pharmacodynamics of macrolides: non-antibiotic pharmacological properties}

Macrolide antibiotics do not only provide clinical benefit in chronic bacterial infections as a result of direct antibacterial effects. They exhibit biofilm inhibiting effects, as well as a variety of immunomodulatory actions that promote host defence (46-49). In epithelial cells, macrolides also inhibit mucus production, through effects on calcium mobilization, associated with changes in extracellular signal-regulated kinase $(\mathrm{ERK}) 1 / 2$ and nuclear factor $\kappa \mathrm{B}(\mathrm{NF \kappa} \mathrm{B})$ signalling pathways (49). In some clinical conditions, including cystic fibrosis (CF) and bronchiolitis obliterans syndrome (BOS), such combined additional actions of macrolides probably make a greater contribution to clinical efficacy than their direct antibacterial actions alone.

Azithromycin is an inhibitor at sub-MIC levels of the bacterial generation of autoinducing, quorum-sensing homoserine lactone (HSL) molecules that regulate bacterial growth and biofilm production $(48,49)$. Modulation of bacterial membrane lipids may be involved in this effect. At similar low concentrations, macrolides also inhibit the production of alginate, the major component of biofilm, probably through inhibition of GDP-mannose dehydrogenase (GMD). The inhibition of biofilm formation increases the susceptibility of the micro-

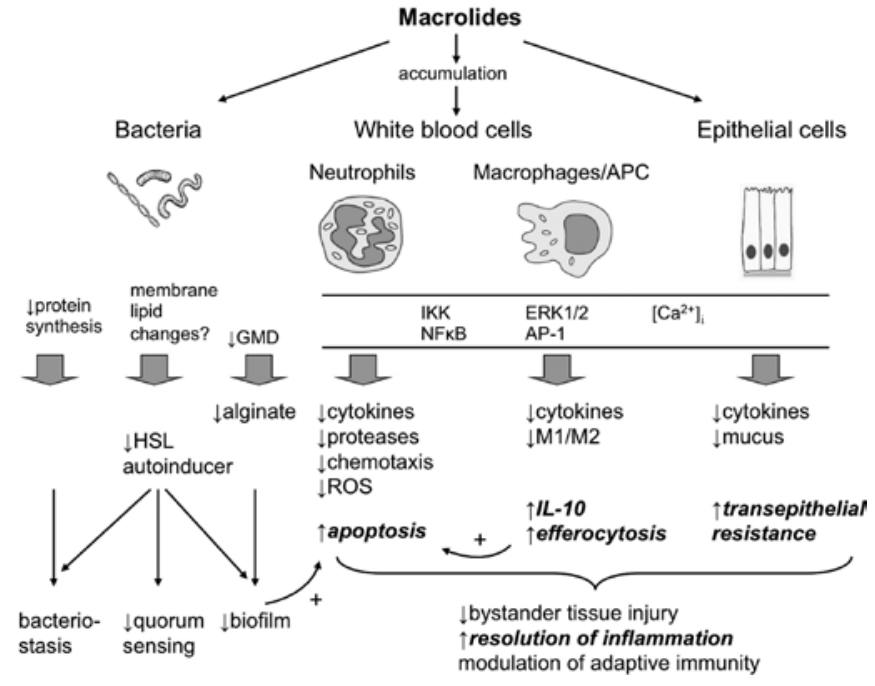

Figure 2. Major mechanisms of action of macrolide antibiotics on processes involved in chronic bacterial infections. In addition to direct bacteriostatic actions, macrolides inhibit quorum-sensing and biofilm formation, which facilitates bacterial clearance by neutrophils. Effects on host cells, including white blood cells, are variously mediated by the inhibition of mitogenactivated protein kinase (MAPK) pathways [particularly extracellular signal-regulated kinase (ERK)1/2], the IкB kinase (IKK) cascade and intracellular calcium increases. Actions of macrolides on macrophages regulate neutrophil functions, including efferocytosis of apoptotic neutrophils. AP-1, activator protein-1; APC, antigen presenting cells; GMD, GDP-mannose dehydrogenase; HSL, homoserine lactone; ROS, reactive oxygen species; $\mathrm{NF} \kappa \mathrm{B}$, nuclear factor $\kappa \mathrm{B}$; IL-10, interleukin-10; M1, classically activated proinflammatory macrophage phenotype; M2, alternatively activated antiinflammatory macrophage phenotype.

organisms to phagocytosis by leukocytes. Such effects are also likely in CBP in which biofilm deposition may occur.

Macrolide antibiotics accumulate several hundred-fold in phagocytic cells and modulate responses of the immune system (Fig. 2) (46,49,50). Azithromycin, a cationic molecule, concentrates particularly in lysosomes. Neutrophils, which have not been exposed to an inflammatory milieu, are initially stimulated by azithromycin, promoting acute antibacterial defence. This has been shown in healthy volunteers treated for 3 days with the drug (51). With continued exposure, macrolides subsequently inhibit a variety of neutrophil and monocyte responses, including inflammatory chemokine and cytokine production, in association with effects on ERK $1 / 2$ and NFKB pathways (Fig. 2) (46,49,50). Macrolides, also promote neutrophil apoptosis, which, not only helps to resolve inflammation, but also facilitates the co-operative killing of bacteria by neutrophils and macrophages (Fig. 2) (52). The inhibitory effects of azithromycin on neutrophil function in vivo are at least partially due to the modulation of macrophage IL- $1 \beta$ production, probably mediated by the inhibition of activator protein-1 (AP-1) expression (53). These actions ameliorate tissue injury and contribute to improved survival in severe bacterial infections.

Following its administration for several weeks or months to patients with chronic inflammatory or infectious conditions, such as chronic obstructive pulmonary disease, azithromycin modifies the type of immune response detected. The classical proinflammatory M1 macrophage phenotype, characterized by interleukin-12 (IL-12) and IL-6 production, is inhibited. Concomitantly, azithromycin promotes the appearance of 
macrophages resembling more the M2 anti-inflammatory phenotype, characterised by IL-10 production and mannose receptor expression, with enhanced phagocytic and efferocytotic (apoptotic cell phagocytosis) capacity (Fig. 2) (54). Similar changes may be observed in monocytes cultured in vitro and in mice infected with $P$. aeruginosa (55).

When studied on classically activated human blood monocytes in vitro, azithromycin inhibited IL-12p70 and proinflammatory chemokine production, without affecting TNF $\alpha$ and enhanced IL-10 expression. NFאB and STAT1 transcription factors in the TLR4 signalling pathway were down-regulated (56). These effects are likely to facilitate the removal of phagocytosed bacteria and the resolution of chronic inflammation, a possibility that may also apply to CBP. The study of the effects of macrolides on markers of mononuclear phagocyte function in chronic prostatitis is noteworthy.

Findings of recent studies suggest that adaptive immune responses to either bacterial or auto-antigens play a role in $\mathrm{CP}$ (57). In this respect, it is worth mentioning that, at least in mice, prolonged macrolide treatment is able to selectively modulate $\mathrm{T}$ cell-mediated immune responses in vivo (58). Clearly, there is much to be gained clinically from investigation of the effects of macrolides on host defence responses in $\mathrm{CP}$.

\section{Macrolide administration: safety issues}

Gastrointestinal disturbances such as diarrhoea, nausea, abdominal pain and vomiting are common as macrolides are capable of increasing GI motility by stimulating motilin (59). Adverse effects of macrolides also include reversible hearing impairment and allergic reactions ranging from urticaria to anaphylaxis. Cholestasis, Stevens-Johnson syndrome and toxic epidermal necrolysis are more rare, but severe adverse effects of this class of antibiotics.

Macrolides, fluoroquinolones as well as other classes of antimicrobial agents have been associated with prolongation of cardiac repolarization. This correlation occurs when these drugs interact with the product of the human Ether-à-go-go Related Gene (KCNH2), coding for the $\mathrm{K}_{\mathrm{v}} 11.1$ potassium channel. Macrolides are capable of producing a blockage of potassium currents in myocyte membranes, resulting in a prolonged QT interval of the electrocardiogram, which may give rise to Torsades de Pointes or polymorphic ventricular tachycardia. Concomitant intake of macrolides and i) type Ia or III anti-arrhythmic agents, ii) non-anti-arrhythmic drugs known to prolong the QT interval, or iii) agents sharing the same metabolic routes as macrolides, may also cause malignant arrythmias (60-62). For example, macrolides are known to be inhibitors of cytochrome CYP3A4. Additionally, inhibition of the CYP3A4-mediated metabolism of cisapride may result in life-threatening cardiotoxicity. Therefore, caution has to be taken in prescribing macrolides in combination with agents that may enhance their cardiotoxic effects. Pharmacological consultation and cardiological work-up are recommended when preparing therapeutic protocols for patients at risk. In general, a corrected QT interval superior to 450 milliseconds or any history of arrhythmias are basic contraindications for initiating macrolide therapy (63).

In addition to their direct cardiotoxic effect, macrolides indirectly enhance digoxin cardiotoxicity. Most authors support macrolide-induced inhibition of digoxin-metabolizing Eubacterium lentum in the gut flora as the key mechanism of this interaction. However, other authors have shown that inhibition of the P-glycoprotein-mediated tubular excretion of digoxin may be a cause of this toxic effect (64). Co-administration of macrolides and digoxin is strongly contraindicated.

\section{Two pivotal macrolide targets in the prostate gland: intracellular bacteria and biofilms}

Aetiological determinants of category II CBP theoretically include a wide range of aerobic and anaerobic bacterial species, yeasts and other cryptic microorganisms (65). Acknowledged pathogens include Escherichia coli, other Enterobacteriaceae, Enterococci (Enterococcus faecalis), Staphylococci (S. aureus and CNS-coagulase negative Staphylococci) and Pseudomonas aeruginosa, but may also include Chlamydia trachomatis and the urogenital Mycoplasmata,Ureaplasma species (U.parvum and U. urealyticum) and Mycoplasma species (M.hominis and M. genitalium) (66). E.coli, Staphylococci and Enterococci may generate bacterial biofilms (67-69), whereas agents such as Ureaplasma spp., Chlamydia trachomatis and Enterococci may infect prostate cells and act intracellularly.

It is generally believed that prostatitis cases refractory to antibacterial therapy may be related to bacterial biofilm formation inside the gland. Studies (68) performed in recent years have demonstrated for the first time the existence of bacterial strains capable of producing biofilms in the prostate. The findings have confirmed the highly aggressive nature of these strains, associated with the expression of other virulence factors and with high antibiotic resistance patterns. These phenomena offer an explanation for the microbiological detection difficulties, symptom persistence and high chemoresistance in CBP patients. Such conditions require long-term treatment regimens, and favour therapy with antibiotics such as macrolides, which show high intracellular accumulation and are capable of interfering with biofilm formation.

Prostatic calcifications, commonly found in CP patients, have also been conclusively associated with calcified bacterial biofilms (68). Categories II CBP and III CP/CPPS may be caused by cryptic, difficult-to-grow bacteria such as Chlamydia trachomatis and the urogenital Mycoplasmata. Electron microscopy, new molecular diagnostic methods for the detection of bacterial nucleic acids and immunological methods able to detect, at the infection site, secretory IgAs specific to these micro-organisms, appear to be the diagnostic techniques of choice for detecting difficult-to-diagnose chronic infections $(68,69)$.

A recent, unpublished, preliminary comparative study of urogenital Mycoplasmata detected by culture and PCR was performed in the STDs centre of a co-author of this article (SM) between September and December 2009. In total ejaculates from $126 \mathrm{CP}$ patients, a $26.6 \%$ prevalence rate for Ureaplasma species, $15 \%$ for Ureaplasma parvum, $6.3 \%$ for $U$. urealyticum and $1.5 \%$ for Mycoplasma hominis were detected. In previous studies on prostatitis by the same group, these microbes were not only present in deep genital tract tissues of men affected by $\mathrm{CP}$, but were also associated with chronic inflammation. This correlation was shown by the presence of proinflammatory cytokines in exposed tissues 
(IL-6, IL-10 and IL-8) (70). Of note, Chlamydia trachomatis is capable of inducing modifications of sperm quality in male individuals affected by CP (71). In a recent study (unpublished data) in which $1161 \mathrm{CP}$ patients (mean age, 36.5 years old) were enrolled, 707 patients were affected by CBP caused by common uropathogens, and 454 patients had $\mathrm{CP}$ associated with $C$. trachomatis prostate infection. Significant statistical differences were reported between groups in terms of sperm concentration, percentage of motile sperm cells, and normal sperm morphology. Correlations between mucosal anti- $C$. trachomatis IgAs, positivity for HSP60, HSP70, sperm concentration and normal morphologic forms were reported. This study demonstrated the contribution of $\mathrm{CP}$, resulting from $C$. trachomatis infection, to the impairment of male fertility, highlighting the probable immune-mediated damage to germinal cells in response to the $C$.trachomatis infection. It may be concluded that a number of immunopathogenetic and biofilm-related phenomena, all mediated by pathogenic bacteria, are involved in CP. The unique combination of high tissue accumulation, anti-microbial, biofilm-inhibitory and immunomodulatory properties of macrolides suggests that they should be particularly effective in CBP.

\section{Clinical evidence of efficacy of macrolides in prostate infections: monotherapy}

Over the last few years, the evidence emerging from a number of clinical studies suggests that macrolides are likely to be a primary therapeutic option for the treatment of CBP.

In a prospective, comparative randomized study, the efficacy and tolerability of azithromycin and ciprofloxacin were compared in the treatment of CP caused by C. trachomatis (72). The authors of this study examined a total of 89 patients with inflammatory findings and the presence of $C$.trachomatis in expressed prostatic secretions (EPS) or in voided bladder urine collected immediately after prostatic massage (VB3). Patients were randomized to receive a total dose of $4.5 \mathrm{~g}$ of azithromycin given as a 3-day 'pulse-dosing' therapy (500 mg once daily for 3 consecutive days each week) for 3 weeks, or ciprofloxacin $500 \mathrm{mg}$ b.i.d. for 20 days. Bacteriological efficacy was evaluated 4-6 weeks after completion of therapy. Significantly higher eradication rates were achieved in the group of patients treated with azithromycin (80\%) vs. the ciprofloxacin group (38.6\%). Similarly, a significantly higher clinical cure rate was achieved in patients treated with azithromycin (68.9\%), when compared to ciprofloxacin (34.1\%, $\mathrm{p}=0.0021)$. The authors concluded that azithromycin and not fluoroquinolones should be the agent of choice in patients with prostatitis caused by $C$. trachomatis, and that both in CBP patients and in patients with inflammatory $\mathrm{CP} /$ CPPS, ciprofloxacin should not be recommended in case of suspected infection by $C$. trachomatis.

These positive results led Skerk et al to optimise dosing regimens for azithromycin in Chlamydial CBP, by comparing the efficacy and tolerability of total doses of 4.5 or $6 \mathrm{~g}$ azithromycin. This trial was performed in the context of a prospective, comparative and randomized study performed on 89 prostatitis patients, randomized to receive a total dose of $4.5 \mathrm{~g}$ of azithromycin administered as a 3-day 'pulse' (500 mg once daily for 3 consecutive days each week) for 3 weeks
(46 patients) or a total dose of $6 \mathrm{~g}$ of azithromycin administered as a 3-day 'pulse' therapy for 4 weeks (43 patients). Eradication rates under the two regimens were not significantly different $(80.43 \%$ patients for the 4.5 -g group vs. $81.40 \%$ for the 6.0-g group) (73). Clinical cure rates also did not significantly differ between total doses of 4.5 or $6.0 \mathrm{~g}$ azithromycin (69.57\% for the $4.5 \mathrm{-g}$ group vs. $72.09 \%$ for the $6.0 \mathrm{-g}$ group). Therefore, shorter regimens are sufficient to effectively eradicate $C$.trachomatis.

The efficacy of azithromycin for treatment of CBP involving $C$. trachomatis was also compared with that of clarithromycin in the context of a comparative study performed on 123 patients (74). A total of 91 per-protocol patients were randomized to receive a total of $4.5 \mathrm{~g}$ of azithromycin $(500 \mathrm{mg}$ once daily for 3 consecutive days each week) for 3 weeks, or $500 \mathrm{mg}$ clarithromycin twice daily. The eradication rates for azithromycin and clarithromycin were high and similar: 80.43 and $80 \%$, respectively ( $\mathrm{p}=0.83$; OR 1.03 ; 95\% CI, 0.33-3.24). The clinical cure rates were $69.57 \%$ for azithromycin and $71.11 \%$ for clarithromycin ( $\mathrm{p}=0.946$; OR -0.93 ; 95\% CI, 0.34-2.51).

Subsequently Skerk et al compared the efficacy of azithromycin and doxycycline in a total of 125 patients with symptoms of CP and with prostate infection caused by $C$. trachomatis. Patients were randomized to azithromycin:doxycycline at a ratio of 2:1. A total of 82 patients received a total dose of $4.0 \mathrm{~g}$ azithromycin administered as a single weekly dose of $1000 \mathrm{mg}$ for 4 weeks and 43 patients received doxycycline $100 \mathrm{mg}$ b.i.d. for 4 weeks. No significant difference was found between the eradication rates achieved by the two antimicrobial agents (azithromycin, 79.3\%; doxycycline, 76.7\%, p=0.82), nor in clinical cure rates between the treatment groups (azithromycin, 68.3\%; doxycycline, 69.8\%, p=0.94) (75). Similar results were obtained in CBP caused by U. urealyticum (76).

Thus, results of the randomized controlled studies described above suggest that azithromycin is a first-choice agent for CBP involving $C$. trachomatis or $U$. urealyticum as causative pathogens. Azithromycin performed better than ciprofloxacin, and showed comparable activity to tetracyclines. A total dose of $4.5 \mathrm{~g}$ azithromycin may be conveniently administered three times weekly in a 'pulse-dosing' manner. This regimen is easy to follow and may result in high patient compliance.

\section{Clinical evidence of efficacy of macrolides in prostate infections: combination therapy}

Azithromycin has been administered within the framework of combination therapy regimens in Chlamydial prostatitis. Results of recent observational studies show that therapy based on the combination of azithromycin and levofloxacin is characterized by high eradication (94\%) and low relapse rates (77).

Other studies have shown that in CBP patients presenting with Chlamydial infection, a combination of azithromycin with different antibacterial agents (rifampicin and pefloxacin) resulted in increased eradication rates, compared to singleagent regimens (78).

Macrolides have been effectively administered in combination with fluoroquinolones for treatment of non-Chlamydial CBP. Observational studies have shown that the combination of azithromycin and ciprofloxacin was characterized by 
Table II. Advantages and disadvantages of macrolide therapy of chronic bacterial prostatitis.

Advantages

Good distribution to the prostate, high PS/PL ratios

High intracellular concentrations

Optimal activity against Chlamydia and genital Ureaplasma

Anti-biofilm activity

Immunomodulatory activity

Biphasic early leukocyte-activating and late inflammation-resolving activity enhances early chemotaxis but favours subsequent rapid removal of immune cells

Good compliance of azithromycin when administered as three times weekly 'pulse-dosing' regimen

General good tolerability

Disadvantages

Activity against $E$. coli or Enterococci is uncertain

Contraindicated in patients at risk of arrhythmias

Contraindicated in combination with agents prolonging the QT EKG interval

May have prokinetic activity in the GI tract

PS, prostate secretions; PL, plasma; E. coli, Escherichia coli.

a high safety profile and was effective in eradicating both traditional uropathogens and unusual pathogens infecting the prostate gland, with similar and high eradication rates (traditional uropathogens, 75-84\%; unusual uropathogens, $82 \%)(79,80)$. Notably, pathogen eradication achieved with an azithromycin-ciprofloxacin combination was characterized by a rapid symptom decrease and by low rates of clinical relapse/ re-infection. This therapeutic combination also decreased the total serum PSA, thus markedly enhancing the cancer detection rate upon biopsy in a patient population (81).

The azithromycin-ciprofloxacin therapeutic protocol was based on administration of azithromycin $(500 \mathrm{mg}$, three times weekly 'pulse-dosing') with ciprofloxacin administered at the once daily dose of $500 \mathrm{mg}$ for 6 weeks. Patients found this original 6-week regimen lengthy and difficult to comply with. In addition, the concentration of fluoroquinolones in prostate secretions is often sub-optimal. The possibility, therefore, was investigated as to whether i) this therapeutic scheme was capable of being modified to administer the same cumulative dose of $21 \mathrm{~g}$ ciprofloxacin at the rate of $750 \mathrm{mg} /$ day for 4 weeks, or ii) the fluoroquinolone dose could be increased to $1000 \mathrm{mg} /$ day (28 g total dose).

Administration of a cumulative dose of $21 \mathrm{~g}$ ciprofloxacin at a rate of $750 \mathrm{mg} /$ day for 4 weeks, as compared to one of $500 \mathrm{mg} /$ day for 6 weeks, increased eradication rates from 62.3 to $77.3 \%$, and the total bacteriological success from 71.8 to $85.6 \%$ (82). Eradication efficacy was further increased when azithromycin was administered with $1000 \mathrm{mg}$ once daily ciprofloxacin (84\%), and notably, was higher than the rate of microbiological eradication $(62 \%)$ with ciprofloxacin monotherapy ( $1 \mathrm{~g} /$ day for 4 weeks). Relapse/re-infection rates in patients receiving the combined regimen were lower, in comparison to those in patients receiving $1 \mathrm{~g} /$ day ciprofloxacin for 4 weeks as a single agent (2.2-fold relapse compared with combination therapy). A marked decrease in pain and voiding signs/symptoms was sustained throughout an 18 month follow-up period. Combination therapy also induced a significant reduction of inflammatory leukocyte counts and serum
PSA, which was more pronounced in the group receiving azithromycin and $750 \mathrm{mg}$ daily doses of ciprofloxacin. Signs and symptoms associated with sexual function (ejaculatory pain, hemospermia and premature ejaculation) were markedly attenuated on microbiological eradication. Of a total of 59 patients showing mild to severe erectile dysfunction (ED) at baseline, 22 had no ED on microbiological eradication nor throughout the follow-up period of 18 months (82).

A recent pilot study was performed to test a 'switchtherapy' protocol, administered to 30 patients showing severe CBP symptoms at baseline and two or more relapse episodes in the previous 12 months. Patients received intravenous azithromycin (500 mg/day) and ciprofloxacin (800 mg/day) for 3 days, followed by oral ciprofloxacin $(1 \mathrm{~g} /$ day $)$ for 25 days. A total of 27 patients $(90 \%)$ showed pathogen eradication at test-of-cure (TOC) visit, persisting in 24 subjects $(80 \%) 6$ months later. At the TOC visit, 25 patients (83\%) showed mild or absent symptoms, measured with the NIH-chronic prostatitis symptom index. These results confirm the efficacy of combined azithromycin and ciprofloxacin, administered via the intravenous route in patients showing severe clinical symptoms (unpublished data).

\section{Conclusions}

Macrolide antibiotics are characterized by an impressive array of distinctive PK and PD properties. These properties include high intracellular accumulation in phagocytes and at sites of infection, including the prostate, broad antibiotic but also biofilm-inhibiting properties, immunomodulating and inflammation-resolving activities. These features offer particular advantages for the treatment of chronic infections of the prostate gland, which are not easily amenable to drug therapy. Macrolides may be exploited to counteract the unsatisfactory rates of clinical symptom improvement and pathogen eradication, a hypothesis that several clinical trials support.

Table II summarizes some of the advantages and disadvantages of macrolide therapy in CBP, as described in this review article, which is based on a symposium on macrolides 
in prostatitis held during the 3rd International Congress on Urogenital and Sexually Transmitted Infections in Opatija, Croatia (May 20-22, 2011). The opinions expressed indicate the consensus among the co-authors that macrolides such as azithromycin are essential tools in the limited armamentarium of physicians and specialists seeking to alleviate the severe burden of $\mathrm{CP}$ infections.

\section{Acknowledgements}

This study was part of the project 'Research on the etiology, epidemiology, diagnostics and treatment of patients with prostatitis syndrome' (principal investigator, V. Škerk) and funded by The Croatian Science Foundation. The authors are thankful to Professor M.J. Parnham, for his careful and insightful revision of the manuscript.

\section{References}

1. Bartoletti R, Cai T, Mondaini N, et al: Prevalence, incidence estimation, risk factors and characterization of chronic prostatitis/ chronic pelvic pain syndrome in urological hospital outpatients in Italy: results of a multicenter case-control observational study. J Urol 178: 2411-2415, 2007.

2. Krieger JN, Nyberg L and Nickel JC: NIH consensus definition and classification of prostatitis. JAMA 282: 236-237, 1999.

3. Litwin MS, McNaughton-Collins M, Fowler FJ, et al: The National Institutes of Health chronic prostatitis symptom index: development and validation of a new outcome measure. Chronic Prostatitis Collaborative Research Network. J Urol 162: 369-375, 1999.

4. Wenninger K, Heiman JR, Rothman I, et al: Sickness impact of chronic nonbacterial prostatitis and its correlates. J Urol 155 965-968, 1996.

5. Cheng I, Witte JS, Jacobsen SJ, et al: Prostatitis, sexually transmitted diseases, and prostate cancer: the California Men's Health Study. PLoS One 5: e8736, 2010.

6. Mahmud SM, Franco EL and Aprikian AG: Use of nonsteroidal anti-inflammatory drugs and prostate cancer risk: a metaanalysis. Int J Cancer 127: 1680-1691, 2010.

7. Jafari S, Etminan M and Afshar K: Nonsteroidal anti-inflammatory drugs and prostate cancer: a systematic review of the literature and meta-analysis. Can Urol Assoc J 3: 323-330, 2009

8. Shao N, Xu B, Mi YY and Hua LX: IL-10 polymorphisms and prostate cancer risk: a meta-analysis. Prostate Cancer Prostatic Dis 14: 129-135, 2011

9. Quintar AA, Doll A, Leimgruber C, et al: Acute inflammation promotes early cellular stimulation of the epithelial and stromal compartments of the rat prostate. Prostate 70: 1153-1165, 2010.

10. Mackern-Oberti JP, Maccioni M, Cuffini C, et al: Susceptibility of prostate epithelial cells to Chlamydia muridarum infection and their role in innate immunity by recruitment of intracellular Toll-like receptors 4 and 2 and MyD88 to the inclusion. Infect Immun 74: 6973-6981, 2006.

11. Gatti G, Rivero V, Motrich RD and Maccioni M: Prostate epithelial cells can act as early sensors of infection by up-regulating TLR4 expression and proinflammatory mediators upon LPS stimulation. J Leukoc Biol 79: 989-998, 2006.

12. Pei Z, Lin D, Song X, et al: TLR4 signaling promotes the expression of VEGF and TGFbeta1 in human prostate epithelial PC3 cells induced by lipopolysaccharide. Cell Immunol 254: 20-27, 2008.

13. Vykhovanets EV, Shukla S, MacLennan GT, et al: Il-1 beta-induced post-transition effect of NF-kappaB provides time-dependent wave of signals for initial phase of intrapostatic inflammation. Prostate 69: 633-643, 2009.

14. Wagenlehner FM, Weidner W and Naber KG: Therapy for prostatitis, with emphasis on bacterial prostatitis. Exp Opin Pharmacother 8: 1667-1674, 2007.

15. Magri V, Perletti G, Bartoletti R, et al: Critical issues in chronic prostatitis. Arch Ital Urol Androl 82: 75-82, 2010.

16. European Association of Urology Urological Infections Guidelines: http://www.uroweb.org/gls/pdf/15_Urological_ Infections.pdf
17. Wagenlehner FM, Naber KG, Bschleipfer T, et al: Prostatitis and male pelvic pain syndrome: diagnosis and treatment. Dtsch Arztebl Int 106: 175-183, 2009.

18. Naber KG and European Lomefloxacin Prostatitis Study Group: Lomefloxacin versus ciprofloxacin in the treatment of chronic bacterial prostatitis. Int J Antimicrob Agents 20: $18-27,2002$.

19. Schaeffer AJ: Clinical practice. Chronic prostatitis and the chronic pelvic pain syndrome. N Engl J Med 355: 1690-1698, 2006.

20. Nickel JC, Patel M and Cameron M: Chronic prostatitis/chronic pelvic pain syndrome: finding a way forward in the United Kingdom: Report from the first United Kingdom symposium on chronic prostatitis. Rev Urol 10: 160-163, 2008.

21. Lipsky BA, Byren I and Hoey CT: Treatment of bacterial prostatitis. Clin Infect Dis 50: 1641-1652, 2010.

22. Jiang SH and Collignon PJ: Treatment of recurrent multiresistant Escherichia coli prostatitis with azithromycin. Med J Aust 194: 208, 2011.

23. Soto SM, Smithson A, Martinez JA, et al: Biofilm formation in uropathogenic Escherichia coli strains: relationship with prostatitis, urovirulence factors and antimicrobial resistance. J Urol 177: 365-368, 2007.

24. Naber KG and Sorgel F: Antibiotic therapy - rationale and evidence for optimal drug concentrations in prostatic and seminal fluid and in prostatic tissue. Andrologia 35: 331-335, 2003.

25. Barza M and Cuchural G: The penetration of antibiotics into the prostate in chronic bacterial prostatitis. Eur J Clin Microbiol 3: 503-505, 1984

26. Perletti G, Wagenlehner FM, Naber KG and Magri V: Enhanced distribution of fourth-generation fluoroquinolones in prostatic tissue. Int J Antimicrob Agents 33: 206-210, 2009.

27. Wagenlehner FM, Weidner W and Naber KG: Therapy for prostatitis, with emphasis on bacterial prostatitis. Expert Opin Pharmacother 8: 1667-1674, 2007.

28. Wagenlehner FM, Kees F, Weidner W, et al: Concentrations of moxifloxacin in plasma and urine, and penetration into prostatic fluid and ejaculate, following single oral administration of $400 \mathrm{mg}$ to healthy volunteers. Int J Antimicrob Agents 31: 21-26, 2008.

29. Foulds G, Madsen P, Cox C, et al: Concentration of azithromycin in human prostatic tissue. Eur J Clin Microbiol Infect Dis 10: 868-871, 1991

30. Giannopoulos A, Koratzanis G, Giamarellos-Bourboulis EJ, et al: Pharmacokinetics of clarithromycin in the prostate: implications for the treatment of chronic abacterial prostatitis. J Urol 165: 97-99, 2001

31. Baumueller A, Hoyme U and Madsen PO: Rosamicin - a new drug for the treatment of bacterial prostatitis. Antimicrob Agents Chemother 12: 240-242, 1977.

32. Carlier MB, Garcia-Luque I, Montenez JP, et al: Accumulation, release and subcellular localization of azithromycin in phagocytic and non-phagocytic cells in culture. Int J Tissue React 16: 211-220, 1994.

33. Ouadrhiri Y, Scorneaux B, Sibille Y and Tulkens PM: Mechanism of the intracellular killing and modulation of antibiotic susceptibility of Listeria monocytogenes in THP-1 macrophages activated by gamma interferon. Antimicrob Agents Chemother 43: 1242-1251, 1999.

34. Van Bambeke F and Tulkens PM: Macrolides: pharmacokinetics and pharmacodynamics. Int J Antimicrob Agents 18: S17-S23, 2001.

35. Drew RH and Gallis HA: Azithromycin - spectrum of activity, pharmacokinetics, and clinical applications. Pharmacotherapy 12: 161-173, 1992.

36. Amsden GW and Gray CL: Serum and WBC pharmacokinetics of $1500 \mathrm{mg}$ of azithromycin when given either as a single dose or over a 3 day period in healthy volunteers. J Antimicrob Chemother 47: 61-66, 2001.

37. Amsden GW, Nafziger AN and Foulds G: Pharmacokinetics in serum and leukocyte exposures of oral azithromycin, 1,500 milligrams, given over a 3- or 5-day period in healthy subjects. Antimicrob Agents Chemother 43: 163-165, 1999.

38. Nightingale $\mathrm{CH}$ : Pharmacokinetics and pharmacodynamics of newer macrolides. Pediatr Infect Dis J 16: 438-443, 1997.

39. Kanatani MS and Guglielmo BJ: The new macrolides. Azithromycin and clarithromycin. West J Med 160: 31-37, 1994.

40. Douthwaite S and Champney WS: Structures of ketolides and macrolides determine their mode of interaction with the ribosomal target site. J Antimicrob Chemother 48 (Suppl T1): 1-8, 2001. 
41. Gaynor M and Mankin AS: Macrolide antibiotics: binding site, mechanism of action, resistance. Curr Top Med Chem 3: 949-961, 2003.

42. Noedl H, Krudsood S, Chalermratana K, et al: Azithromycin combination therapy with artesunate or quinine for the treatment of uncomplicated Plasmodium falciparum malaria in adults: a randomized, phase 2 clinical trial in Thailand. Clin Infect Dis 43: 1264-1271, 2006.

43. Gilbert DN, Moellering RC, Eliopoulos GM and Sande MA (eds): The Sanford Guide to Antimicrobial Therapy. 41st edition Antimicrobial Therapy Inc., Sperrivylle, VA, pp67-72, 2011.

44. Vester B and Douthwaite S: Macrolide resistance conferred by base substitutions in $23 \mathrm{~S}$ rRNA. Antimicrob Agents Chemother 45: 1-12, 2001.

45. Tenson T and Mankin AS: Short peptides conferring resistance to macrolide antibiotics. Peptides 22: 1661-1668, 2001.

46. Čulić O, Eraković V and Parnham MJ: Anti-inflammatory effects of macrolide antibiotics. Eur J Pharmacol 429: 209-229, 2001.

47. Parnham MJ: Immunomodulatory effects of antimicrobials in the therapy of respiratory tract infections. Curr Opinion Infect Dis 18: 125-131, 2005 .

48. Tateda K, Ishii Y, Kimura S, et al: Suppression of Pseudomonas aeruginosa quorum-sensing systems by macrolides: a promising strategy or an oriental mystery? J Infect Chemother 13: 357-367, 2007.

49. Kanoh S and Rubin BK: Mechanisms of action and clinical application of macrolides as immunomodulatory medications. Clin Microbiol Rev 23: 590-615, 2010.

50. Eraković Haber V: Makrolidi - više od antibiotika. Infektol glasn/Croat J Infect 31: 29-39, 2011.

51. Culić O, Eraković V, Cepelak I, et al: Azithromycin modulates neutrophil function and circulating inflammatory mediators in healthy human subjects. Eur J Pharmacol 450: 277-289, 2002.

52. Silva MT: Macrophage phagocytosis of neutrophils at inflammatory/infectious foci: a cooperative mechanism in the control of infection and infectious inflammation. J Leuk Biol 89: 675-683, 2011.

53. Bosnar M, Čužić S, Bošnjak B, et al: Azithromycin inhibits macrophage interleukin-1 $\beta$ production through inhibition of activator protein-1 in lipopolysaccharide-induced murine pulmonary neutrophilia. Int Immunopharmacol 11: 424-434, 2011.

54. Hodge S, Hodge G, Jersmann H, et al: Azithromycin improves macrophage phagocytic function and expression of mannose receptor in chronic obstructive pulmonary disease. Am J Respir Crit Care Med 178: 139-148, 2008.

55. Feola DJ, Garvy BA, Cory TJ, et al: Azithromycin alters macrophage phenotype and pulmonary compartmentalization during lung infection with Pseudomonas. Antimicrob Agents Chemother 54: 2437-2447, 2010.

56. Vrančić M, Banjanac M, Nujić K, et al: Azithromycin distinctively modulates classical activation of human monocytes in vitro. Br J Pharmacol: Jul 4, 2011 (E-pub ahead of print).

57. Hou Y, DeVoss J, Dao V, et al: An aberrant prostate antigenspecific immune response causes prostatitis in mice and is associated with chronic prostatitis in humans. J Clin Invest 119: 2031-2041, 2009

58. Iveti Tkalčevi V, Čuži S, Dominis Kramari M, et al: Topical azithromycin and clarithromycin inhibit acute and chronic skin inflammation in sensitized mice, with apparent selectivity for Th2-mediated processes in delayed type hypersensitivity. Inflammation: Feb 19, 2011 (E-pub ahead of print).

59. Weber FH Jr, Richards RD and McCallum RW: Erythromycin a motilin agonist and gastrointestinal prokinetic agent. Am J Gastroenterol 88: 485-490, 1993.

60. Iannini PB: Cardiotoxicity of macrolides, ketolides and fluoroquinolones that prolong the QTc interval. Expert Opin Drug Saf 1: 121-128, 2002.

61. Guo D, Cai Y, Chai D et al: The cardiotoxicity of macrolides: a systematic review. Pharmazie 65: 631-640, 2010

62. Nahata M: Drug interactions with azithromycin and the macrolides: an overview. J Antimicrob Chemother 37 (Suppl C): 133-142, 1996
63. Friedlander AL and Albert RK: Chronic macrolide therapy in inflammatory airways diseases. Chest 138: 1202-1212, 2010.

64. Hughes J and Crowe A: Inhibition of P-glycoprotein-mediated efflux of digoxin and its metabolites by macrolide antibiotics. J Pharmacol Sci 113: 315-324, 2010.

65. Mazzoli S: Conventional bacteriology in prostatitis patients: microbiological bias, problems and epidemiology on 1686 microbial isolates. Arch Ital Urol Androl 79: 71-75, 2007.

66. Cai T, Mazzoli S, Bartoletti R, et al: May Chlamydia trachomatis be an aetiological agent of chronic prostatitis infection? Chronic prostatitis due to Chlamydia trachomatis infection: a challenge for the urologist. Andrologia 43: 84, 2011.

67. Nickel JC and McLean RJC: Bacterial biofilms in urology. Infect Urol 11: 169-175, 1998.

68. Mazzoli S: Biofilms in chronic bacterial prostatitis (NIH-II) and in prostatic calcifications. FEMS Immunol Med Microbiol 59: 337-344, 2010.

69. García-Castillo M, Morosini MI, Galvez M, et al: Differences in biofilm development and antibiotic susceptibility among clinical Ureaplasma urealyticum and Ureaplasma parvum isolates. J Antimicrob Chemoth 62: 1027-1030, 2008.

70. Mazzoli S, Cai T, Rupealta V, et al: Interleukin 8 and antiChlamydia trachomatis mucosal $\mathrm{Ig} \mathrm{A}$ as urogenital immunologic markers in patients with $C$. trachomatis prostatic infection. Eur Urol 51: 1385-1393, 2007.

71. Mazzoli S, Cai T, Addonisio P, et al: Chlamydia trachomatis infection is related to poor semen quality in young prostatitis patients. Eur Urol 57: 708-714, 2010.

72. Skerk V, Schönwald S, Krhen I, et al: Comparative analysis of azithromycin and ciprofloxacin in the treatment of chronic prostatitis caused by Chlamydia trachomatis. Int J Antimicrob Agents 21: 457-462, 2003.

73. Skerk V, Krhen I, Lisić M, et al: Azithromycin: 4.5- or 6.0-gram dose in the treatment of patients with chronic prostatitis caused by Chlamydia trachomatis-a randomized study. J Chemother 16: 408-410, 2004

74. Skerk V, Schönwald S, Krhen I, et al: Comparative analysis of azithromycin and clarithromycin efficacy and tolerability in the treatment of chronic prostatitis caused by Chlamydia trachomatis. J Chemother 14: 384-389, 2002.

75. Skerk V, Krhen I, Lisić M, et al: Comparative randomized pilot study of azithromycin and doxycycline efficacy in the treatment of prostate infection caused by Chlamydia trachomatis. Int J Antimicrob Agents 24: 188-191, 2004.

76. Skerk V, Mareković I, Markovinović L, et al: Comparative randomized pilot study of azithromycin and doxycycline efficacy and tolerability in the treatment of prostate infection caused by Ureaplasma urealyticum. Chemotherapy 52: 9-11, 2005.

77. Magri V, Marras E, Skerk V, et al: Eradication of Chlamydia trachomatis parallels symptom regression in chronic bacterial prostatitis patients treated with a fluoroquinolone-macrolide combination. Andrologia 42: 366-375, 2010.

78. Smelov V, Gorelov A, Smelova N and Krylova T: Single-drug or combined antibacterial therapy in the treatment of patients with chronic prostatitis and Chlamydia trachomatis? Int J Antimicrob Agents 23: S83-S87, 2004.

79. Magri V, Trinchieri A, Pozzi G, et al: Efficacy of repeated cycles of combination therapy for the eradication of infecting organisms in chronic bacterial prostatitis. Int $\mathbf{J}$ Antimicrob Agents 29: 549-556, 2007.

80. Magri V, Trinchieri A, Ceriani I, et al: Eradication of unusual pathogens by combination pharmacological therapy is paralleled by improvement of signs and symptoms of chronic prostatitis syndrome. Arch Ital Urol Androl 79: 93-98, 2007.

81. Magri V, Trinchieri A, Montanari E, et al: Reduction of PSA values by combination pharmacological therapy in patients with chronic prostatitis: implications for prostate cancer detection. Arch Ital Urol Androl 79: 84-92, 2007.

82. Magri V, Montanari E, Skerk V, et al: Fluoroquinolone-macrolide combination therapy for chronic bacterial prostatitis: retrospective analysis of pathogen eradication rates, inflammatory findings and sexual dysfunction. Asian J Androl: doi: 10.1038/aja.2011.36 (In press). 